\title{
Comparison of isokinetic strengthening with functional strengthening of lower limb and their effect on gait in hemiparesis due to stroke.
}

\author{
Shalini Singhal, Monalisa Pattnaik, Patitapaban Mohanty \\ Swami Vivekanand National Institute of Rehabilitation Training and Research, Olatpur, Bairoi, Cuttack, India
}

\begin{abstract}
Background: A large number of survivors of stroke present with various disabilities including motor deficits characterized by weakness (hemiparesis) on one side of the body opposite to the site of lesion. Muscle weakness in the affected lower limb of stroke patients is a major factor inhibiting gait ability which is the most frequently stated goal by persons with stroke. Progressive resistance strength training has been found to strengthen paretic muscles after stroke including isokinetic strengthening and functional strengthening. Purpose: The present study intended to examine whether one of the two types of lower limb strengthening (isokinetic strengthening and functional strengthening) is more beneficial in improving peak torque, six-minute walk test and step length in post stroke hemiparetic persons. Method: A total 30 number of subjects were randomly selected and assigned to either of the two groups after meeting the inclusion and exclusion criteria. All participants underwent an initial baseline assessment. Both groups received conventional physiotherapy. Group 1 in addition, received isokinetic strengthening, whereas group 2 in addition, received functional strengthening for a period of 6 weeks, 3 days/ week. All participants received a follow up assessment after completion of 6 weeks. Results: The overall results of the study suggest that after 6 weeks of intervention both the groups (isokinetic strengthening and functional strengthening) improved significantly in peak torque of hip flexor, hip extensor, knee flexor, knee extensor, ankle dorsiflexor, plantarflexor, six-minute walk test, step length affected and step length non-affected. Isokinetic strengthening group showed significantly better improvement in peak torque of hip flexor, hip extensor, knee flexor, knee extensor and six-minute walk test. Conclusion: Isokinetic strengthening of lower limb is more effective than functional strengthening in improving peak torque of hip-knee flexor and extensor muscles and six-minute walk test. However, no significant difference was noted in peak torque of ankle muscles and step length of both affected and non-affected side in post stroke hemiparetic persons.
\end{abstract}

Keywords: Isokinetic strengthening, Functional strengthening, Stroke, Hemiparesis/hemiplegia, Gait.

Accepted on April 12, 2017

\section{Introduction}

Stroke can be characterized as an interruption of the blood supply to the brain or hemorrhage into the brain tissue, commonly involving a disruption in the motor and sensory pathways [1]. The World Health Organization (WHO) defines stroke as "rapidly developing clinical signs of focal (or global) disturbance of cerebral function, lasting more than 24 hours or leading to death, with no apparent cause other than that of vascular origin" [2].

A large number of survivors of stroke present with various disabilities, including impairment of sensory, motor, mental, perceptual and language functions. The motor deficits are characterized by paralysis (hemiplegia) or weakness (hemiparesis) on one side of the body opposite to the site of lesion [3]. This weakness is due to reduced activity of lower limb muscles. There is reduction in muscle fiber number and increase fatigability and decreased as well as altered motor unit recruitment which results in type II fibres atrophy in weakened muscles. Stroke subjects likely develop lower oxidative capacity along with impaired neural drive the rate of torque development is more deteriorated in paretic muscles [4-6]. Weakness is recognized as a limiting factor in the motor rehabilitation of patients after stroke. Bobath advocated that weakness in an agonist was attributable to spastic restraint imposed by the antagonist muscle group [7].

Post-stroke hemiplegic gait is a mixture of deviations and compensatory motion dictated by residual functions. [8]. Muscle weakness and paralysis, poor motor control and soft tissue contracture are major contributors to walking dysfunction after stroke. The spatiotemporal adaptations include: decreased walking speed, short or uneven step and stride lengths, increased stride width, increased double support phase, dependence in support through the hands with assistive devices. [9] Improved walking function is the most frequently stated goal by persons with stroke. For this reason, gait retraining is an important focus in the rehabilitation of persons with stroke. Despite this effort to regain optimal walking function, only a small percentage attains a walking speed that is required for community activities (Figures 1 and 2).

Muscle weakness in the affected lower limb of stroke patients 


\section{Peak Torque Hip Flexors}

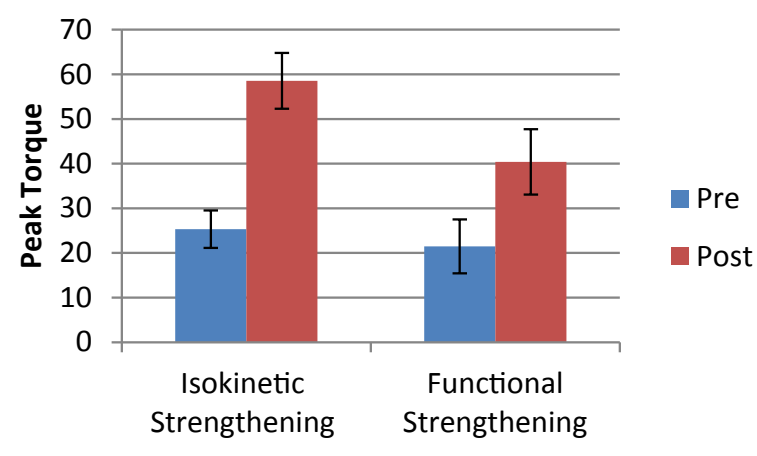

Figure 1. Peak torque hip flexors.

\section{Peak Torque Hip Extensors}

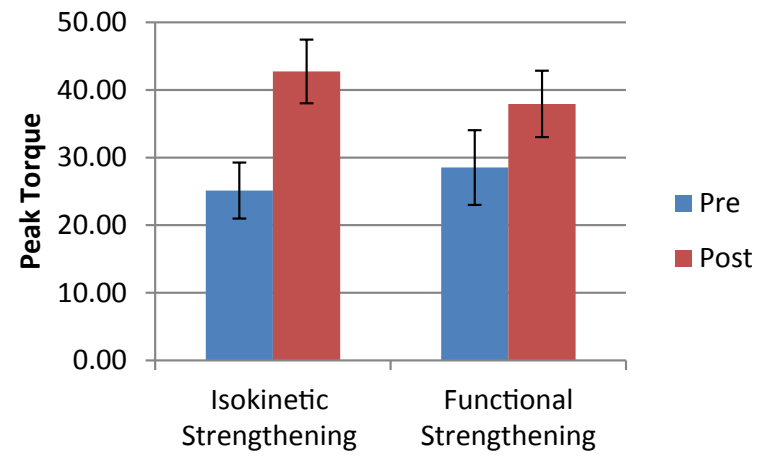

Figure 2. Peak torque-hip extensors.

is a major factor inhibiting gait ability [10]. Isometric exercise, isotonic exercise, and isokinetic exercise are used as resistance exercises to increase muscle strength [11,12]. Isokinetic exercise is a method that enables the muscle to exert maximum strength within the exercise range of all joints at a constant speed [13]. Various exercise methods are performed with the purpose of strengthening the muscles in the affected lower limb of stroke patients, and isokinetic exercise has been proposed as an effective exercise method [14].

Progressive resistance strength training has been found to strengthen paretic muscles after stroke [15-17]. Randomized controlled trials (RCTs) using strength training as intervention have shown inconsistent results regarding the effects on gait speed and sit-to-stand activities [16,18,19]. The effect of strength training on functional activities for patients in the subacute phase has only been examined in a few RCTs showing inconsistent results $[15,19,20]$.

EMG studies have shown the importance of hip joint flexors, hip joint extensors, knee joint flexors, knee joint extensors, ankle joint dorsiflexors and plantarflexors in lower limb activities [21]. Hip flexors, knee flexors (Figure 3) and ankle dorsiflexors works during sitting down, swing phase of gait, stepping up to lift foot from stair surface and swing forward whereas hip extensors, knee extensors (Figure 4) and ankle plantarflexors works during standing up, stance phase of gait and stepping up to lower the foot on next stair and to lift body up. Eng and Winter reported that the flexion/extension forces of the hip, knee, and ankle accounted for $82 \%$ of the total work over a
Peak Torque Knee Flexors

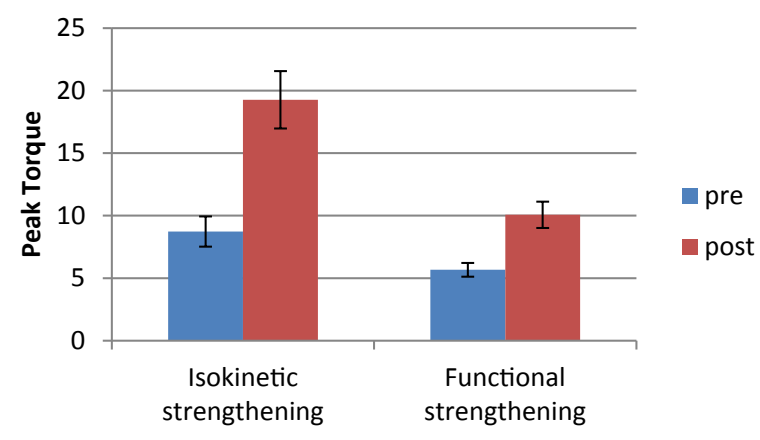

Figure 3. Peak torque-knee flexors.

\section{Peak torque knee extensors}

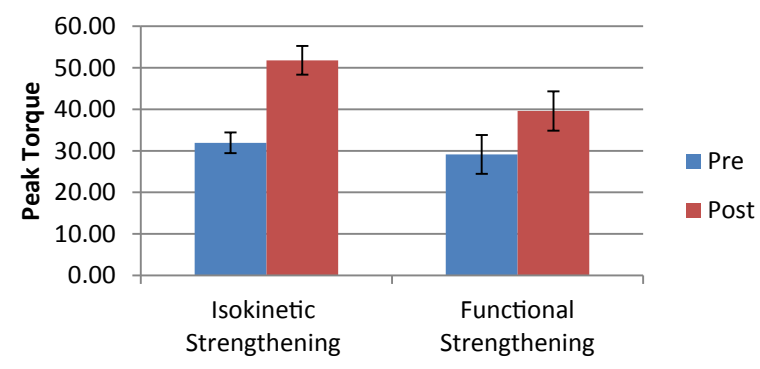

Figure 4. Peak torque-knee extensors.

stride as opposed to $15 \%$ and $3 \%$ in the frontal and transverse planes, respectively [22].

Isokinetic exercise is a method that enables the muscle to exert maximum strength within the exercise range of all joints at a constant speed. Six weeks of maximal isokinetic strengthening of the knee musculature, $[10,23] 9$ weeks of bilateral isokinetic program using the Kinetron [24], as well as an 8-week isokinetic strengthening program of isolated flexor and extensor muscle groups of the paretic lower extremity [25] has also been reported to improve walking speed (Figure 5).

Functional strengthening on the other hand is beneficial for the development of functional abilities. The functional strength training program is designed to facilitate appropriate power in the weak muscles of the affected leg in graded activities or sequences of activities. Most of the exercises are weightbearing. In various studies, functional strength training of the

\section{Peak torque ankle dorsiflexors}

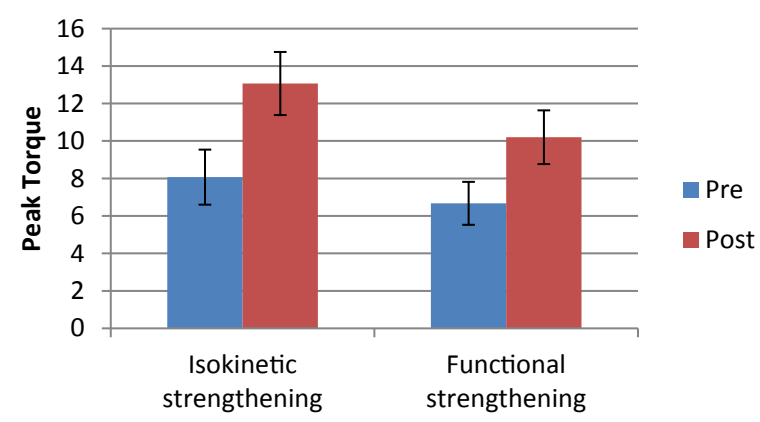

Figure 5. Peak torque-ankle dorsiflexors. 
legs was found to be more effective than traditional training to improve weight-bearing on the affected side and gait speed $[17,26]$. Functional strength training of the affected leg improved subacute stroke patients' ability to perform functional activities more than usual training with no particular focus on improving strength.

Due to paucity in studies comparing the two strengthening techniques, the aim of this study was to find out whether one of these two forms of strengthening exercises will provide better result on gait of hemiparetic individuals.

\section{Aim of the study}

To examine whether one of the two types of lower limb strengthening performed with isokinetic strengthening and functional strengthening is more beneficial in improving peak torque, six-minute walk test and step length in post stroke hemiparetic persons.

\section{Methodology}

\section{Study design}

Experimental two groups, pre-test, post-test structured study design.

Subjects/Participants: A total of 30 subjects with hemiparesis due to stroke were recruited randomly. The average age of subjects was $51.27 \pm 8.47$ with a range of 38 to 66 years. Group 1 -isokinetic strengthening group-15 subjects (13 males and 2 females), group 2-functional strengthening group-15 subjects (13 males and 2 females). The age of subjects in isokinetic strengthening group ranged from 38-66 years with the mean age $47.93 \pm 8.08$ whereas the age of subjects in functional strengthening group ranged from 41-64 years with the mean age $54.60 \pm 7.72$. The duration of stroke ranged from 4-18 months in isokinetic strengthening group and 5-21 months in functional strengthening group with the mean duration of stroke $10.27 \pm$ 4.49 and $11.87 \pm 5.04$ respectively (Figure 6).

Research setting: The study was conducted in neurorehabilitation section of the Physiotherapy department of Swami Vivekanand national institute of rehabilitation training and research (SVNIRTAR).

Inclusion criteria: Both male and female, both right and left hemiplegics, first time onset of stroke, duration of stroke ranged from 3 months to 2 years, clinically stable, spasticity of major muscle groups with modified Ashworth scale of less than 2, able to walk independently with or without orthosis (Figures 7 and 8).

Exclusion criteria: Contractures in lower limb, any cognitive deficit in stroke patients, other neurological and musculoskeletal conditions, people with cardiovascular diseases, recurrent stroke, participants who could not comprehend and obey verbal commands.

Instrumentation: CSMI HUMAC/NORMTM isokinetic device, measuring tape calibrated from $0 \mathrm{~cm}$ to $150 \mathrm{~cm}$ to measure the floor distance for 6-minute walk test, stopwatch (a cellphone application used in flight mode) to measure the time for 6-minute walk test, paper roll to measure step length, ink to measure step length, step stool $-10 \mathrm{~cm}, 14 \mathrm{~cm}, 18 \mathrm{~cm}, 6$-minute walk test, step-length.

\section{Peak torque ankle plantarflexors}

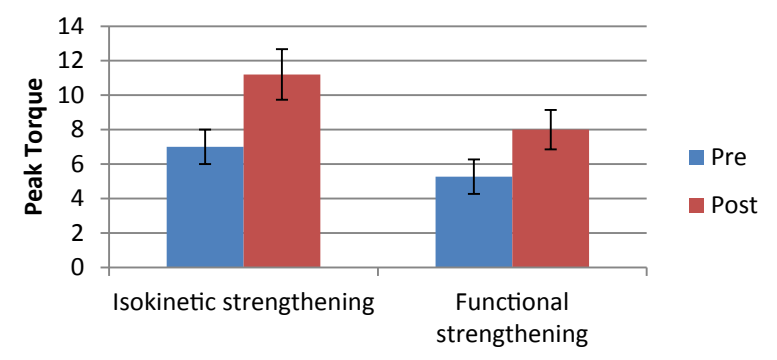

Figure 6. Peak torque-ankle plantar flexors.

\section{Six Minute Walk Test}

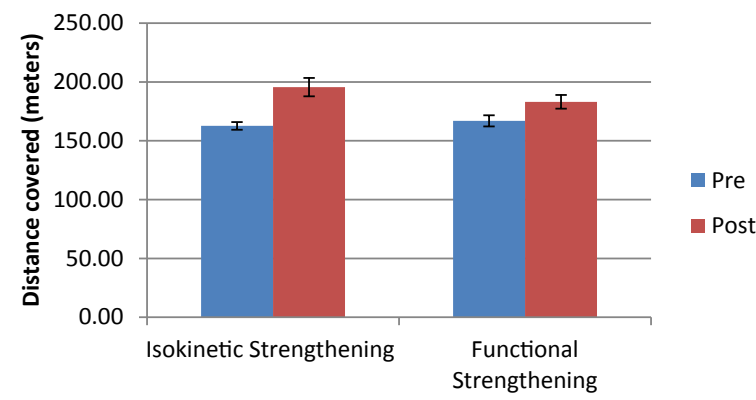

Figure 7. Six minute walk test.

\section{Step Length Affected}

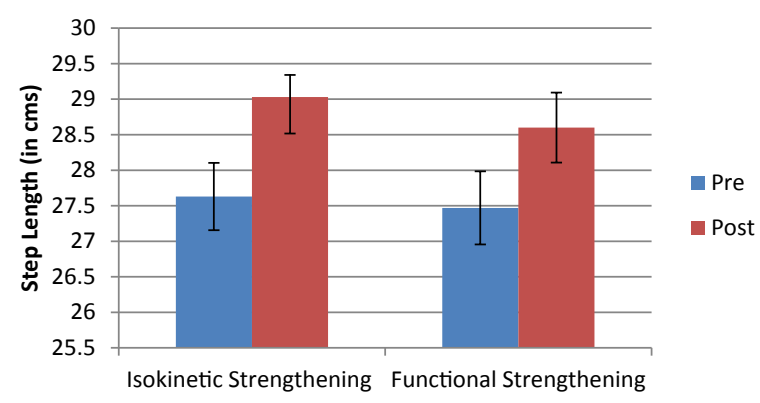

Figure 8. Step-length affected.

\section{Outcome measure:}

1. Peak torque as measured by isokinetic device: 5 repetitions at $60 \%$ angular velocity was done for concentric contraction of hip flexor/extensor, knee flexor/extensor and ankle plantarflexor/dorsiflexor [27].

2. 6-minute walk test: The distance travelled by the subject in 6-minutes was calculated [28,29].

3. Step length of both affected and non-affected side: Foot prints method of gait analysis was used with help of ink and paper roll. Distance between initial contact of the one foot to another foot for middle 5 steps were evaluated on a 10 -meter roll [30].

\section{Procedure}

After meeting the inclusion and exclusion criteria through an assessment proforma, informed consent was taken and subjects were randomly allocated to either of the two groups. 


\section{Step length Non- Affected}

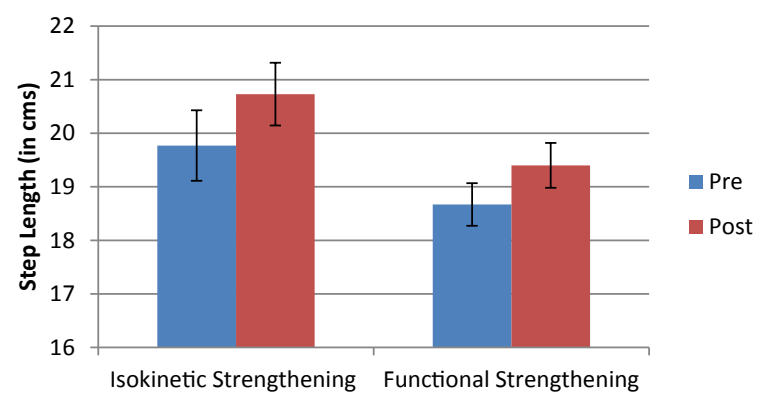

Figure 9. Step length non-affected. significantly more improvement with isokinetic strengthening group as compared to functional strengthening group in hip flexor/extensor and knee flexor/extensor group of muscles $(\mathrm{p}<0.05)$. Note that in ankle dorsiflexor/plantarflexor there was no significant difference between the groups. Graph 1-6 summarizes improvement in peak torque of each muscle group from pre to post in both groups.

\section{Six-minute walk test}

Six-minute walk test improved with both isokinetic strengthening and functional strengthening exercises, however the improvement was significantly more in isokinetic strengthening group as compared to functional strengthening group $(\mathrm{p}<0.05)$. Graph 7 illustrates the improvement from pre to post in both the groups (isokinetic strengthening and functional strengthening).

\section{Step length}

For both isokinetic strengthening group and functional strengthening group, the step length of both affected and non-affected side were higher after the intervention than at baseline however, there was no significant difference between the groups. Graph 8-9 illustrates the improvement from pre to post of both the groups (isokinetic strengthening and functional strengthening) in step length of affected and non-affected side with no significant difference between the groups.

\section{Discussion}

The overall results of the study suggest that after 6 weeks of intervention both the groups (isokinetic strengthening and functional strengthening) improved significantly from pre to post treatment in peak torque of hip flexor, hip extensor, knee flexor, knee extensor, ankle dorsiflexor, plantarflexor, six minute walk test, step length affected and step length nonaffected. Isokinetic strengthening group showed significantly better improvement in peak torque of hip flexor, hip extensor, knee flexor, knee extensor and six-minute walk test.

\section{Peak torque}

In this study, both strengthening groups showed significant improvement in hip extensor, hip flexor, knee extensor, knee flexor, ankle dorsiflexor and ankle plantarflexor peak torque from pre to post readings after 6 weeks of therapy. Isokinetic strengthening group showed better improvement in hip extensor, hip flexor, knee extensor and knee flexor peak torque as compared to functional strengthening group. The increase in the peak torque in hip flexor, hip extensor, knee flexor, knee extensor, ankle dorsiflexor and ankle plantarflexor group of muscles in isokinetic strengthening group was $131.07 \%$, $70.03 \%, 120.73 \%, 62.23 \%, 61.96 \%$ and $60 \%$ whereas in functional strengthening group was $88.17 \%, 32.95 \%, 77.60 \%$, $35.94 \%, 52.92 \%$ and $51.80 \%$ respectively.

The improvement in strength in both groups may be contributed to the effect of conventional exercises which may be due to the learning and practice effect. Various studies have shown improvement in strength in control group in which conventional exercises were given $[32,33]$.

There improvement in isokinetic strengthening group from pre 
to post measurements might be due to improvement in motor unit recruitment and firing and motor learning [23,33]. Various studies [34-37] found that the strength improvement seen in a 6 -week training program can be attributed to improvement in neural activation rather than muscle hypertrophy.

It has been shown in various studies previously that isokinetic strengthening can result in increased peak torque in adults after stroke. Kim et al. found improved strength in hip, knee and ankle muscles of paretic limb ranging from $7 \%$ to $155 \%$ with 6 weeks of concentric isokinetic strength training at $60 \% \mathrm{~s}$ angular velocity [18]. Sharp et al. also found relative gains in strength ranging from $15.8 \%$ to $153.9 \%$ of knee muscles after isokinetic training of 6 weeks at 30, 60 and $120 \%$ s angular velocity [23]. The knee extensor showed greater improvement as compared to knee flexor muscles which was attributed to improved motor unit recruitment. Similar result was shown by Engardt and Keerthi et al. $[10,33]$. Nakamura et al. found eight weeks after training the strength of affected side explained $50 \%$ of the variance, becoming twice as large as that at the start of training. [38] Sen et al. also showed improvement in peak torque with isokinetic strengthening [39]. All these results were in agreement with present study.

In present study, the functional strengthening group received various lower limb functional activities thrice a week for 6 weeks and showed an increase in pre to post isokinetic peak torque measurement of affected lower limb. The increase in peak torque might be due to accordance with the specificity of training principle as functional movements are specific in nature. When the functional training program includes the element of strength training, the training improves the outcome of muscle strength [40]. Bedi et al. in their study showed that less intense but task specific training regimens can produce cortical reorganization which may be responsible for improvement in strength [41]. Cress et al. found functional strength training for 1 year in older women results in change in myofibrillar density and area in type IIb fiber which was associated with strength improvement [42]. In this study only 6 weeks of training was given which might have resulted in little change in myofibrillar density.

In this study, all of the exercises that are incorporated were concentrated on promoting loading of lower limbs. This additional body weight loading over lower limbs explains the mean increment in strength of lower limb muscles. This result is consistent with the study done by Shaik et al. [31]. Bale et al. showed improvement in strength with functional strength training for 5 days/week for 4 weeks [26]. Yang et al. also showed improvement in strength with functional strength training of about $10.1 \%$ to $77.9 \%$ (average $41.1 \%$ ) on the paretic side muscle groups by giving 4 weeks functional strength training thrice a week [17].

In this study, the isokinetic strength training showed better improvement as compared to functional strength training group in some of the muscle groups which may be because isokinetic exercise device provides a suitable mechanical means of maintaining the maximal muscular force of a body segment throughout the range of motion without permitting acceleration [43]. Engardt in their study told that isokinetic device allows training within a large movement range and supply resistance to the patients' maximal voluntary, contractile tension within the whole range of movement [10]. Perrine found that the intensive loading through the isokinetic training might activate previously dormant fibers [44]. Bale showed in their study that less improvement in functional strength training group may be contracted to the challenge of keeping dose of training high enough [26]. These all reasons may have contributed to greater improvement in isokinetic strength training group as compared to functional strengthening.

Improvement in peak torque of ankle plantarflexor and dorsiflexor was not significant between the two groups in this study. Milot also showed lack of significant difference between the groups which can be related to the variability of the response to training programs [45]. Hakkinen showed that not all muscle groups have a similar rate of strength development and this combined with the pretraining status of individuals could partly explain the variability in individual training responses [46]. Also in the lower limb, studies have been done to a lesser extent in peri-articular muscles of the ankle.

\section{Six-minute walk test}

In this study both strengthening groups showed significant improvement in six-minute walk test from pre to post readings after 6 weeks of therapy. Isokinetic strengthening group showed better improvement as compared to functional strengthening group. The isokinetic strengthening group showed $20.29 \%$ whereas functional strengthening showed $9.70 \%$ improvement in six-minute walk test with strengthening.

According to Perry, the sequence of muscle action relates to three major tasks being accomplished during each gait cycle: weight acceptance, single limb support, and limb advancement [47]. Preparation of the limb for weight acceptance begins in terminal swing when the hip extensors and quadriceps are activated. Following initial floor contact, the loading response is an increase in the intensity of these hip and knee extensor muscles to stabilize the trunk and limb against the rapid transfer of body weight. To ensure knee stability, the hamstrings and single joint hip extensors exchange their intensities. Continuation of the swing phase ankle dorsiflexor control provides a heel rocker for progression and shock absorbing knee flexion. Ankle plantar flexion modulates the heel rocker to protect the quadriceps from over demand. In this study, there is increase in muscle activity of all these group muscles which may be responsible for increase in gait speed and endurance.

There was significant improvement in isokinetic strengthening group from pre to post measurements in this study. Concentric isokinetic strengthening was given at $60 \%$ s angular velocity thrice a week for 6 weeks. Common gait problems in hemiplegia arise due to various reasons which may also include unequal step length, and weak muscles of hip, knee and ankle [48]. Studies have shown improvement in gait performance with strength gains in spastic conditions. Damiano et al. and Horvat showed improvement in gait with resistance exercise in children with spastic cerebral palsy $[49,50]$. EMG study done by Dubo et al. showed that knee extensor is active concentrically in majority of gait cycle [51]. Flansjberg showed that knee extensor is considered as an important parameter for walking 
endurance [52]. Along with this, knee flexor, hip flexor and ankle plantarflexor are also associated with walking endurance. As there was improvement in peak torque of all these muscle groups with isokinetic strengthening that may have contributed to improvement in six-minute walk test. Slower voluntary muscle reaction time was seen after 6 weeks of isokinetic training in healthy individuals which suggests that isokinetic training improves motor task performance [34].

Various other studies also showed a high correlation between isokinetic torque of paretic knee and locomotor function in stroke patients. Sen et al. showed improvement in 6-minute walk test with maximal concentric isokinetic strength training in 50 subjects 5 days a week for 3 weeks [39]. Sharp et al. and Kim et al. also showed improvement in gait velocity with isokinetic strengthening $[18,23]$. The results are in agreement with present study.

Functional strengthening group also showed improvement in six-minute walk test with 6 weeks of training. Yang et al. showed that the task-oriented progressive resistance strength training programme can improve lower extremity strength which is significantly associated with gain in gait parameters [17]. Bale showed that the great attention and exerted effort during training of weight-bearing activities seem to have enhanced muscular control of the affected leg and symmetry between the legs in functional activities which may have helped in improvement of 6-minute walk test [26].

Yang et al. showed an improvement in 6-minute walk test with 4 weeks task oriented progressive resistance strength training to hemiparetic individuals [17]. Similar studies were shown by Bale, Salbach et al. and Shaik $[26,31,53]$. These all results are in agreement with this study and showed improvement in gait with functional strength training.

In this study, isokinetic strengthening groups showed more improvement in six-minute walk test as compared to functional strengthening group. Thistle et al. and Smith et al. showed that that isokinetic exercise increased performance levels more efficiently than other exercise modes [54,55]. It was shown previously by Flansjberg that knee extensor is considered as an important parameter for walking endurance [52]. In this study, there was better improvement in knee extensor strength with isokinetic strengthening as compared to functional strengthening. This increase in strength may have contributed to better improvement of 6-minute walk test in isokinetic strengthening group in the present study.

\section{Step length}

In this study both groups (isokinetic strengthening and functional strengthening) showed improvement in step length in both affected and non-affected side from pre to post measurements. There was no significant difference noted between the groups. The increase in the step length of affected and non-affected side in isokinetic strengthening group was $5.07 \%$ and $4.85 \%$ whereas in functional strengthening group was $4.11 \%$ and $3.91 \%$ respectively.

Forward foot placement with respect to the trunk and trunk progression contributes to step-length and is responsible for asymmetry of step length in stroke patients as was shown by Roerdink [56]. Balasubramanian found that reduced trunk progression during paretic stance may reflect an impaired propulsion generating capacity of the paretic leg [57]. The reason behind improvement of step length in affected and non-affected limb with strength training in this study might be generation of increased propulsion capacity of the paretic leg as well as non-paretic leg which may have increased trunk progression. Along with this, the paretic leg swing may be restricted as well as found by Olney [58]. It is possible that because of an impaired swing initiation by the hip flexors or an excessive flexion synergy decreased step length results in paretic limb. So, an improvement in hip flexor strength may be responsible for improvement in step length in affected limb in this study. The improvement in step length in non-affected limb may be due to increase in weight bearing ability in affected limb and allowing better propulsion of non-affected limb.

Isokinetic strengthening group showed improvement in step length from pre to post in both affected and non-affected side. This improvement in step length may be contributed to increase in strength of knee extensor muscle. Nakamura et al. showed that concentric knee extensor contraction during deceleration phase is determinant of walking velocity and step length [59]. Olawale et al. and Wilder showed improvement in step length on affected side with isokinetic strengthening $[24,60]$. They attributed this improvement to the increase in muscular strength of the affected lower limbs as a result of the training regimen.

In present study improvement was seen in step length from pre to post measurements in both affected and non-affected side with functional strength training. This may be due to the gross improvement of strength which may have improved the functional activities irrespective of the technique applied [17]. Shaik found improvement in step length with weight bearing task oriented exercise in 30 hemiparetic individuals [31]. They attributed this increase in step length to increase in hip extension in late stance phase because these changes are associated with moving the trunk forward over the stance foot, thus providing the hip flexors with better mechanical advantage to generate power to pull-off the limb, resulting in a larger contra lateral step length and an increase in speed. Vats and Park et al. also found improvement in step length with step up exercises and stair climbing exercises respectively $[32,61]$. The results of all those studies are in agreement with present study.

The significant difference was not found in both groups (isokinetic strengthening and functional strengthening) in step length. This may be due to low specificity of training in both the groups. Also, Park reported that, increased step length because of increased dorsiflexion, contributed to the prevention of foot drop and lower limb stability upon initial contact in the swing phase and explained that a stable walking pattern could enhance both static and dynamic stability and in turn increase step length [62]. In this study, there was no significant difference in improvement of peak torque of ankle muscle, this may be the reason for absence of significant difference in step length improvement in both the groups. 


\section{Conclusion}

Isokinetic strengthening of lower limb is more effective than functional strengthening in improving peak torque of hip-knee flexor and extensor muscles and six-minute walk test. However, no significant difference was noted in peak torque of ankle muscles and step length of both affected and non-affected side in post stroke hemiparetic persons.

\section{Limitations}

Study sample was small, no control group was taken, carry over effect of the study has not been studied.

\section{Recommendations for Future Studies}

Studies with larger sample size should be performed, follow up study should be done to see the long-term effect, study with isokinetic strengthening of both lower limbs can be done.

\section{Clinical Utility}

Isokinetic strengthening of lower limb should be incorporated in exercise protocol in clinical settings for hemiparetic individuals due to stroke in order to improve their strength and gait parameters which can enhance their quality of life.

\section{References}

1. Toole JF. Vascular diseases. Etiology and pathogenesis. In: Rowland LP, editor. Merrit's textbook of neurology. Philadephia: Lea and Febiger. 1984;145-53.

2. Aho K, Harmsen P, Hatano S, et al. Cerebrovascular disease in the community: Results of a WHO collaborative study. Bull World Health Organ. 1980;58:113-30.

3. Dietz V, Ketelsen UP, Berger W, et al. Motor unit involvement in spastic paresis. Relationship between leg muscle activation and histochemistry. J Neurol. 1993;33:109-14.

4. Scelsi R, Lotta S, Lommi G, et al. Hemiplegia atrophy. Morphology findings in the anterior tibial muscles of patients with cerebral vascular accidents. Acta Neuropathol. 1984;62:324-31.

5. Hachisuka K, Umezu Y, Ogata H. Disuse muscle atrophy of lower limbs in hemiplegic patients. Arch Phys Med Rehabil. 1997;78:13-8.

6. Bourbonnais D, Vanden Noven S. Weakness in patients with hemiparesis. Am J Occup Ther. 1989;43:313-19.

7. Bobath B. Adult hemiplegia: Evaluation and treatment. $2^{\text {nd }}$ edition. London: Heinemann Medical Books Ltd. 1978.

8. Balaban B, Tok F. Gait Disturbances in Patients with Stroke. PMR. 2014;6(7):635-42.

9. Carr JH, Shepherd R, Butterworth H. Stroke rehabilitation guidelines for exercise and training to optimize Motor Skill; First edition. 2003;8(4):261.

10. Engardt M, Knutsson E, Jonsson M, et al. Dynamic muscle strength training in stroke patients: Effect on knee extension torque, electromyographic activity and motor function. Arch Phys Med Rehabil. 1995;76:419-25.
11. Scharf HP, Eckhardt R, Maurus M, et al. Metabolic and hemodynamic changes during isokinetic muscle training. A controlled clinical trial. Int J Sports Med. 1994;15:S56-9.

12. Knapik JJ, Ramos MU. Isokinetic and isometric torque relationships in the human body. Arch Phys Med Rehabil. 1980;61:64-7.

13. Davis GJ. Eccentric isokinetics. Orthop Phys Ther Clin. 1992;1:297-336.

14. Chon JS, Shin JS, Chun SI. The effectiveness of isokinetic exercise for hemiplegic patients. J Korean Acad Rehab Med. 1991;15:67-70.

15. Inaba M, Edberg E, Montgomery J, et al. Effectiveness of functional training, active exercise, and resistive exercise for patients with hemiplegia. Phys Ther. 1973;1:28-35.

16. Ouellette MM, LeBrasseur NK, Bean JF, et al. Highintensity resistance training improves muscle strength, self-reported function, and disability in longterm stroke survivors. Stroke. 2004;35:1404-09.

17. Yang Y, Wang R, Lin K, et al. Task-oriented progressive resistance strength training improves muscle strength and functional performance in individuals with stroke. Clin Rehabil. 2006;20:860-70.

18. Kim CM, Eng JJ, MacIntyre D, et al. Effects on isokinetic strength training on walking in persons with stroke: A double blind controlled pilot study. J Stroke Cerebrovasc Dis. 2001;10:265-73.

19. Giuliani C, Light KE, Rose D. The effect of an isokinetic exercise program on the performance of sit-to-stand in patients with hemiparesis. Proceedings: Forum on Stroke Rehabilitation. 1992;4:49-54.

20. Moreland JD, Goldsmith $\mathrm{CH}$, Huijbregts $\mathrm{MP}$, et al. Progressive resistance strengthening exercises after stroke: A single-blind randomized controlled trial. Arch Phys Med Rehabil. 2003;84:1433-40.

21. He H, Kiguchi K, Horikawa E. A study on lower-limb muscle activities during daily lower-limb motions. Int J Bioelectromagn. 2007;9(2):79-84.

22. Eng JJ, Winter DA. Kinetic analysis of the lower limb during walking: What information can be gained from a three-dimensional model? J Biomech. 1995;28:753-8.

23. Sharp SA, Brouwer BJ. Isokinetic strength training of the hemiparetic knee: Effects on function and spasticity. Arch Phys Med Rehabil. 1997;78:1231-6.

24. Wilder PA, Sykes J. Using an isokinetic exercise machine to improve the gait pattern in a hemiplegic patient. Phys Ther. 1982;62:1291-5.

25. Karimi H. Doctoral dissertation. Isokinetic strength training and its effect on the biomechanics of gait in subjects with hemiparesis as a result of stroke. Queen's University; Kingston, Canada. 1996.

26. Bale M. Does functional strength training of the leg in subacute stroke improve physical performance? A pilot randomized controlled trial. Clin Rehabil. 2008;22:911-21. 
Citation: Singhal S, Pattnaik M, Mohanty P. Comparison of isokinetic strengthening with functional strengthening of lower limb and their effect on gait in hemiparesis due to stroke. J Neurol Neurorehabil Res. 2017;2(1):46-54.

27. Eng JJ, Kim CM, MacIntyre DL. Reliability of lower extremity strength measures in persons with chronic stroke. Arch Phys Med Rehabil. 2002;83(3):322-8.

28. Eng JJ, Dawson AS, Chu KS. Submaximal exercise in persons with stroke: test-retest reliability and concurrent validity with maximal oxygen consumption. Arch Phys Med Rehabil. 2004;85(1):113-8.

29. Flansbjer UB, Holmback AM, Downham D, et al. Reliability of gait performance tests in men and women with hemiparesis after stroke. J Rehabil Med. 2005;37(2):75-82.

30. Pardo V, Knuth D, McDermott B, et al. Validity, reliability and minimum detectable change of the maximum step length test in people with stroke. J Neurol sci. 2013;325(12):74-8.

31. Shaik R, Shabnam S. A study to know the effectiveness of task oriented progressive resistance strength training on lower extremity strength and functional performance in patients with chronic stroke. Asian J Health Med Res. 2015;01(1):14-7.

32. Vats M. Efficacy of task specific step-up exercises on the gait parameters of chronic hemiparetic stroke individuals. Int J Physiother Res. 2013;04:130-7.

33. Keerthi CS, Madhavi K, Srikumari V, et al. Efficacy of isokinetic strength training and balance exercises on lower limb muscles in subjects with stroke. Int J Physioth Res. 2013;02:25-9.

34. Wojtys EM, Huston LJ, Taylor PD, et al. Neuromuscular adaptations in isokinetic, isotonic, and agility training programs. Am j sports med. 1996;24(2):187-92.

35. Hakkinen K, Komi PV. Electromyographic changes during strength training and detraining. Med Sci Sports Exerc. 1983;15:455-60.

36. Moritani T, DeVries HA. Neural factors versus hypertrophy in time course of muscle strength gain. Am J Phys Med. 1979;58:115-30.

37. Sale DG. Neural adaptation to resistance training. Med Sci Sports Exerc. 1988;20:S135-45.

38. Nakamura R, Watanabe S, Handa T, et al. The relationship between walking speed and muscle strength for knee extension in hemiparetic stroke patients: A follow-up study. Tohoku J exp Med. 1988;154:111-3.

39. Sen SB, Ozbudak DS, Timur E, et al. Effects of the bilateral isokinetic strengthening training on functional parameters, gait, and the quality of life in patients with stroke. Int J Clin Exp Med. 2015;8(9):16871-9.

40. Liu CJ, Shiroy DM, Jones LY, et al. Systematic review of functional training on muscle strength, physical functioning, and activities of daily living in older adults. Eur Rev Aging Phys Act. 2014;11:95-106.

41. Bedi PK, Arumugam N, Kanimozhi. Context and task related approach in rehabilitation after stroke: A systematic review. Int J Physiother Res. 2014;2(1):312-7.
42. Cress ME, Conley KE Function, Functional Training: Muscle Structure, and performance in Older Women. JOSPT. 1996;24.

43. Hislop HJ, Perrine JJ. The isokinetic concept of exercise. Phys Ther. 1967;47:114-117.

44. Perrine JJ. Isokinetic exercise and the mechanical potentials of muscle. JOHPER. 1968;5:40-5.

45. Milot MH, Nadeau S, Gravel D, et al. Gait performance and lower-limb muscle strength improved in both upper-limb and lower-limb isokinetic training programs in individuals with chronic stroke. Hindawi Publishing Corporation ISRN Rehabilitation Article. 2013;929758:10.

46. Hakkinen K. Factors influencing trainability of muscular strength during short term and prolonged training. NSCA J. 1985;7(2):32-7.

47. Perry J. Normal Gait. Atlas of limb prosthetics: Surgical, prosthetic, and rehabilitation principles. Rosemont, IL, Am Acad Ortho Surg. 2002;13.

48. Sadeghi H, Allard P, Duhaine M. Contributions of lower muscle power in gait of people without impairments. Phys Ther. 2000;80:1188-96.

49. Damiano DL, Vaughan CL, Abel MF. Muscle response to heavy resistance exercise in children with spastic cerebral palsy. Dev Med Child Neurol. 1995;37:731-9.

50. Horvat M. Effects of a progressive resistance training program on an individual with spastic cerebral palsy. Am Corrective Ther J. 1987;41:7-11.

51. Dubo HIC, Peat M, Winter DA et al. Electromyographic temporal analysis of gait: Normal human locomotion. Arch phys Med Rehabil. 1976;57:415-20.

52. Flansbjer UB, Downham D, Lexell J. Knee muscle strength, gait performance, and perceived participation after stroke. Arch Phys Med Rehabil. 2006;87:974-80.

53. Salbach NM, Mayo NE, Wood-Dauphinee S, et al. A taskorientated intervention enhances walking distance and speed in the first year post stroke: a randomized controlled trial. Clin Rehabil. 2004;18(5):509-19.

54. Thistle HG, Hislop HJ, Moffroid MT, et al. Isokinetic contraction: A new concept of resistive exercise. Arch Phys Med Rehabil. 1967;48:279-82.

55. Smith MJ, Melton P. Isokinetic versus isotonic variableresistance training. Am J Sports Med. 1981;19:275-9.

56. Roerdink M, Beek PJ. Understanding inconsistent steplength asymmetries across hemiplegic stroke patients: Impairments and compensatory gait. Neurorehabil Neural Repair. 2011;25(3):253-8.

57. Balasubramanian CK, Bowden MG, Neptune RR, et al. Relationship between step length asymmetry and walking performance in subjects with chronic hemiparesis. Arch Phys Med Rehabil. 2007;88:43-9. 
58. Olney SJ, Griffin MP, McBride ID. Temporal, kinematic, and kinetic variables related to gait speed in subjects with hemiplegia: A regression approach. Phys Ther. 1994;74:872-85.

59. Nakamura R, Hosokawa T, Tsuji I. Relationship of muscle strength for knee extension to walking capacity in patients with spastic hemiparesis. Tohoku J exp Med. 1985;145(3):335-40.

60. Olawale OA, Akinfeleye AM. Effects of strengthening of lower limb muscle groups on some gait parameters in adult patients with stroke. J Nigeria soc physiother. 2002;14(2):70-4.

61. Park KH, Kim DY, Kim TH. The effect of step climbing exercise on balance and step length in chronic stroke patients. J Phys Ther Sci. 2015;27:3515-8.

62. Park YH, Kim YM, Lee BH. An ankle proprioceptive control program improves balance, gait ability of chronic stroke patients. J Phys Ther Sci. 2013;25:1321-4.

\section{*Correspondence to:}

Patitapaban Mohanty

Swami Vivekanand National Institute of Rehabilitation

Training and Research

Olatpur, Cuttack

India

Tel: +919437487139

E-mail: ppmphysio@rediffmail.com 